\title{
Projection-Based Image Registration in the Presence of Fixed-Pattern Noise
}

\author{
Stephen C. Cain, Majeed M. Hayat, Senior Member, IEEE, and Ernest E. Armstrong
}

\begin{abstract}
A computationally efficient method for image registration is investigated that can achieve an improved performance over the traditional two-dimensional (2-D) cross-correlation-based techniques in the presence of both fixed-pattern and temporal noise. The method relies on transforming each image in the sequence of frames into two vector projections formed by accumulating pixel values along the rows and columns of the image. The vector projections corresponding to successive frames are in turn used to estimate the individual horizontal and vertical components of the shift by means of a one-dimensional (1-D) cross-correlation-based estimator. While gradient-based shift estimation techniques are computationally efficient, they often exhibit degraded performance under noisy conditions in comparison to cross-correlators due to the fact that the gradient operation amplifies noise. The projection-based estimator, on the other hand, significantly reduces the computational complexity associated with the 2-D operations involved in traditional correlation-based shift estimators while improving the performance in the presence of temporal and spatial noise. To show the noise rejection capability of the projection-based shift estimator relative to the 2-D cross correlator, a figure-of-merit is developed and computed reflecting the signal-to-noise ratio (SNR) associated with each estimator. The two methods are also compared by means of computer simulation and tests using real image sequences.
\end{abstract}

Index Terms-Fixed-pattern noise suppression, image registration, motion estimation, vector projection.

\section{INTRODUCTION}

$\mathbf{I}$ MAGE registration is commonly used to provide electronic stabilization of sensors or to facilitate the processing of multiple motion-compensated frames in order to improve image quality (e.g., by means of temporal averaging). Some new applications of registration also include resolution enhancement from multiple frames of data [1]-[5] and nonuniformity correction in focal-plane arrays [1], [6]-[8]. In many situations, the performance of algorithms that employ image registration information relies heavily on the accuracy of the shift estimates. The accuracy of the shift estimates generated using various registration algorithms is, in turn, strongly affected by the temporal and spatial

Manuscript received July 7, 2000; revised August 30, 2001. This work was supported by the National Science Foundation (CAREER Program MIP-9733308). The associate editor coordinating the review of this manuscript and approving it for publication was Dr. Christoph Stiller.

S. C. Cain is with the Department of Electrical and Computer Engineering, University of Dayton, Dayton, OH 45469-0245 USA and also with the Department of Engineering, Purdue University at Fort Wayne, Fort Wayne, IN 46805-1499 USA.

M. M. Hayat is with the Electro-Optics Program and the Electrical and Computer Engineering Department, University of Dayton, Dayton, $\mathrm{OH}$ 45469-0245 USA and also with the Electrical and Computer Engineering Department, The University of New Mexico, Albuquerque, NM 87131-1356 USA (e-mail: hayat@eece.umn.edu; mhayat@udayton.edu).

E. E. Armstrong is with OptiMetrics, Inc., AFRL/SNJM, Wright Patterson

AFB, OH 45433-7700 USA (e-mail: ernest.armstrong@sn.wpafb.af.mil).

Publisher Item Identifier S 1057-7149(01)10571-3. noise inherent in all sensors [9]. Temporal noise, which fluctuates randomly from frame to frame, includes photo-detection noise, background or stray light, dark current, read-out noise, etc. In contrast to temporal noise, spatial noise has the characteristic that its realizations do not vary from frame to frame resulting in an ambient spatial pattern which superimposes itself on the true scene [10]. This spatial noise is often referred to as fixed-pattern noise and is due to the fact that every detector in the array responds differently to light. Fixed-pattern noise is especially undesirable in image registration since any pattern that remains stationary in time will tend to bias any shift-estimation algorithm toward solutions that favor no shifts between the frames of image data. Fixed-pattern noise can be reduced by calibrating the sensor by means of imaging target scenes with uniform intensities. Fixed-pattern noise can also be reduced from sequences of video by post-processing algorithms [6], [7], [11]. However, some of these methods inherently rely on accurate image registration of the raw video [8]. It is therefore very desirable to have a registration algorithm that is tolerant to fixed-pattern noise.

A commonly used registration technique is based on maximizing the two-dimensional (2-D) cross-correlation function between successive frames, in which case, the shift estimate is defined as the maximizing point [12]. The 2-D cross-correlator is generally regarded as a robust estimator in the presence of noise in comparison to more computationally-efficient estimators. Unfortunately, its implementation involves three discrete-Fourier transforms (DFTs) at the size of the image. Gradient-based shift estimation techniques [2], [13], on the other hand, have a reduced computational complexity but are sensitive to fixed-pattern noise since they may measure false spatial gradients that will be consistently present in successive frames.

In this paper, the concept of integral projection is employed to develop a projection-based registration technique that is computationally efficient and can achieve improved performance over the traditional 2-D cross-correlation-based techniques in the presence of both fixed-pattern and temporal noise. This concept was first conceived from Huang's observation that the linear phase associated with simple translations is encoded on the Fourier axes [14]. Since then the integral projection shift-estimation technique had been introduced to help accomplish inter-frame image encoding by registering subblocks within an image with subblocks within successive frames in a video sequence [15]-[17]. Under the method considered in this paper, each image in a sequence is transformed into two vector projections, formed by accumulating pixel values along the rows and columns of the image, which are subsequently used to estimate the horizontal and vertical components of the shift by means of a one-dimensional (1-D) cross-correlation-based 
estimator. All of the 2-D DFTs associated with the traditional cross-correlator are therefore replaced by 1-D operations. Most importantly, by virtue of the row/column averaging mechanism involved in the projections and the mutual statistical independence of the response characteristics of the detectors, the effect of fixed-pattern noise can be significantly reduced in many practical situations. Although this 1-D technique for image registration has been used in other similar applications [15]-[17], the notion that the projection operation improves performance over the 2-D correlator in the presence of either temporal or fixed-pattern noise has been either completely unknown or not well understood. It is the goal of this paper to reveal the mechanism by which this improved performance is realized and provide a mathematical framework by which the performance improvement can be understood and quantified.

This paper is organized as follows. In Section II, a description of the projection-based estimator is given and an explanation is presented as to why the projections preserve the shift information. In Section III, a signal-to-noise ratio (SNR) analysis is performed that compares the performance of the 2-D cross-correlation shift estimator to that of the projection-based shift estimator in the presence of both temporal and fixed-pattern noise. A performance figure-of-merit is defined for both shift estimators and evaluated using two image models that reflect scenarios when the image possesses high and low degrees of statistical spatial correlation. In Section IV, computer simulations are generated demonstrating the performance of the projection technique on simulated image sequences. In Section V, real image sequences are used to demonstrate the performance of the projection technique. The main conclusions are summarized in Section VI.

\section{The Projection-BAsed ShIFT Estimator}

Consider observing two successive measurements (frames) of a scene $i$ at two distinct times. The time between the two frames is assumed to be sufficiently short so that the scene remains unchanged and the sensor motion results in only a small relative translation of the scene. Let $d_{1}$ denote the first measurement represented by the $N \times N$ matrix whose elements are

$$
d_{1}(x, y)=i(x, y)+q_{1}(x, y)
$$

where $q_{1}$ is a general zero-mean random noise process (temporal and/or spatial) associated with the first frame. Suppose that in the second frame, the scene $i$ is translated by an amount $(\alpha, \beta)$ in the horizontal and vertical directions so that the second frame is given by

$$
d_{2}(x, y)=i(x-\alpha, y-\beta)+q_{2}(x, y)
$$

where $q_{2}$ is the zero-mean random noise associated with the second frame, and the shifts $\alpha$ and $\beta$ are assumed to be integers. Before we introduce the projection-based estimator, we review a commonly-used (based on the 2-D cross-correlation) approach for estimating $\alpha$ and $\beta$ given the two frames $d_{1}$ and $d_{2}$. The technique relies on maximizing the sample 2-D cross-correlation defined by

$$
C(z, w) \triangleq \sum_{x=1}^{N} \sum_{y=1}^{N} d_{1}(x, y) d_{2}(z+x, w+y)
$$

where the shifts are assumed for convenience to be circular. (Border effects resulting from the circular shift become negligible as $N$ increases.) In particular, the traditional 2-D crosscorrelation shift estimator selects the shift estimates, $\left(\hat{\alpha}_{1}, \hat{\beta}_{1}\right)$, by the rule

$$
\left(\hat{\alpha}_{1}, \hat{\beta}_{1}\right) \triangleq \arg \max _{z, w} C(z, w)
$$

In contrast to (2), the projection-based estimation procedure is divided into two distinct operations:

1) the projection operation, which is performed by summing up pixels in the rows or columns of the image;

2) performing the $1-D$ cross-correlation maximization on the projections.

More precisely, let the projections of the image, $d_{i}, i=1,2$, in the horizontal and vertical directions be defined, respectively, by

$$
d_{i}^{x}(y) \triangleq \sum_{x=1}^{N} d_{i}(x, y)
$$

and

$$
d_{i}^{y}(x) \triangleq \sum_{y=1}^{N} d_{i}(x, y)
$$

The vertical and horizontal vector cross-correlation functions are then given by

$$
P_{y}(z)=\sum_{x=1}^{N} d_{1}^{y}(x) d_{2}^{y}(z+x)
$$

and

$$
P_{x}(w)=\sum_{y=1}^{N} d_{1}^{x}(y) d_{2}^{x}(w+y)
$$

Using these statistics, the horizontal and vertical shifts, respectively, are estimated individually using

$$
\hat{\alpha_{2}} \triangleq \arg \max _{z} P_{y}(z)
$$

and

$$
\hat{\beta}_{2} \triangleq \arg \max _{w} P_{x}(w)
$$

We now show that in the absence of noise [i.e., $d_{1}(x, y)=$ $i(x, y)$ and $\left.d_{2}(x, y)=i(x-\alpha, y-\beta)\right]$, the projection-based estimator is equivalent to the 2-D cross-correlator. First, note that a straightforward calculation shows that the vertical projections $d_{1}^{y}(x)$ and $d_{2}^{y}(x)$ can be written as

$$
d_{1}^{y}(x)=\frac{1}{N} \sum_{u=0}^{N-1} I(u, 0) e^{j 2 \pi(u x) / N}
$$

and

$$
d_{2}^{y}(x)=\frac{1}{N} \sum_{u=0}^{N-1} I(u, 0) e^{j 2 \pi u(x-\alpha) / N}
$$

respectively, where $I(u, v)$ is the DFT of the image $i(x, y)$. We now substitute the above projections into the expression for the 
projection-based shift estimator given in (3) and apply the convolution property of the DFT to obtain

$$
P_{y}(z)=\frac{1}{N} \sum_{u=0}^{N-1} I(u, 0) I^{*}(u, 0) e^{-j 2 \pi(z-\alpha) u / N} .
$$

One can immediately recognize $I(u, 0) I^{*}(u, 0)$ as the DFT of the auto-correlation of $d_{1}^{y}$, which is defined by

$$
R_{11}^{y}(z) \triangleq \sum_{x=1}^{N} d_{1}^{y}(z+x) d_{1}^{y}(x) .
$$

Finally, after some straightforward algebra it can be shown that $P_{y}(z)=R_{11}^{y}(z-\alpha)$. Since any auto-correlation is at a maximum at zero [18], the maximum of $P_{y}$ occurs when $z=\alpha$, which implies that $\hat{\alpha_{2}}=\alpha$. This shows that the projection-based shift estimator correctly locates the shift in the horizontal direction without noise and with the assumption that all shifts are circular (no new information enters the scene). A similar argument can be used to show that $\hat{\beta}_{2}=\beta$.

In any real imaging system, the scene does not shift circularly, which causes new information to enter the field of view. This new information is similar to noise, which makes the Fourier arguments for accurate image registration less valid. The projection-based estimator is sensitive to new information entering the scene and so the following noncircular correlation estimate is developed with the projections as opposed to a circular correlation estimate. In order to help the projection-based shift estimation algorithm ignore new information entering the scene, we introduce the window function $w_{f}$ that has the following spatial structure: $w_{f}(x)=1$ if $\left|x-m_{p}\right| \leq\left(N / 2-\delta_{s}\right)$ and $w_{f}(x)=0$ otherwise. In the inequality, $m_{p}$ is the spatial midpoint of the horizontal projection and $\delta_{s}$ is the maximum shift or upper bound of the displacement in pixels between to successive image frames.

We use the window function in a modified correlation function shown below for $|z| \leq \delta_{s}$, where $\delta_{s}$ is the maximum displacement expected in the image sequence

$$
\begin{aligned}
P_{y}(z)=\sum_{x=1}^{N} d_{1}^{y}(x) w_{f}(z+x) & d_{2}^{y}(z+x) \\
& -\left(\sum_{j=1}^{N} d_{1}^{y}(j) w_{f}(z+j) / N\right) \bar{d}_{2}^{y} .
\end{aligned}
$$

In this equation, $\bar{d}_{2}^{y}$ is the average value of this projection. Similarly, the projection in the vertical direction is formed in the following manner. The shift estimate is determined from (3) by an exhaustive search over all integers $|z|<\delta_{s}$. The window function is applied to the projection in the other dimension in the same way. In Section III, the performance of the projection-based shift estimator will be compared analytically to that of the 2-D cross-correlation shift estimator in the presence of both temporal and fixed-pattern noise.

\section{Performance Analysis}

For purposes of this study, two image models will be investigated. The first model addresses cases when the image lacks spatial correlation and the second model addresses cases when

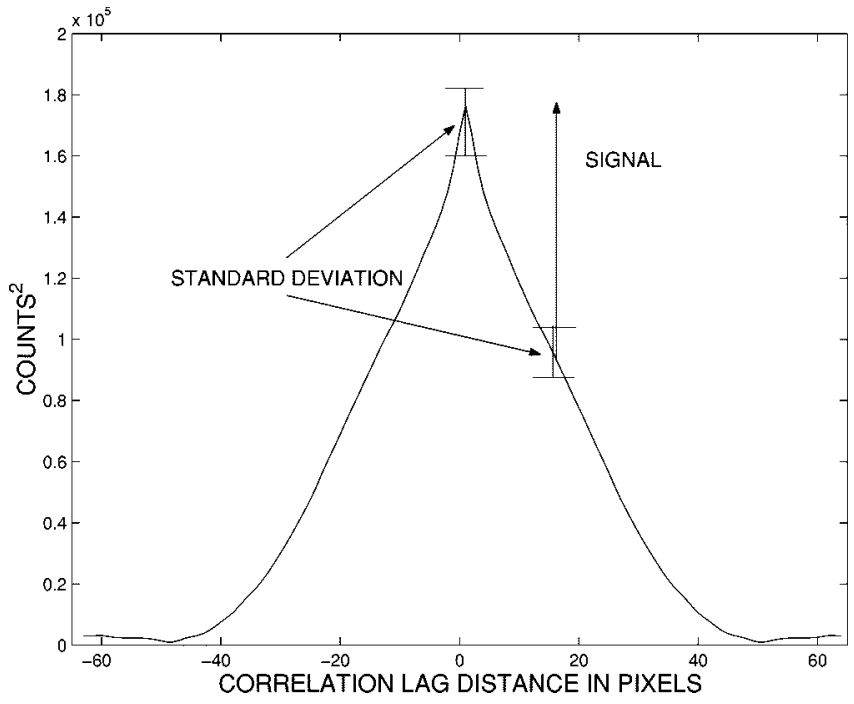

Fig. 1. Mean of the cross-correlation function for $(\alpha, \beta)=(0,0)$, with the standard deviation of the correlation function shown as an error bar. The signal is taken as the difference between the peak amplitude and some off-peak value while the noise is taken as the sum of the variances at the peak and the off-peak positions.

the image exhibits a high degree of spatial correlation. We begin by developing a figure-of-merit, which quantifies the performance of the shift estimators.

In order to motivate the design of a meaningful figure-ofmerit, we first overview the uncertainty in the shift estimates. Observe that for the 2-D cross-correlation shift estimator, errors in the shift estimation occur when the noise causes false peaks in the cross-correlation of the observed image. Fig. 1 shows the amplitude of a typical 1-D cross-correlation function (noise-free) as a function of the correlation shift. If a position other than the peak of the noiseless cross-correlation function is to be selected as the shift estimate, the net effect of noise at the true and false positions in the sample cross-correlation must exceed the difference between the peak of the noise-free cross-correlation at the true shift (i.e., at zero) and the values at off-peak positions, as shown in Fig. 1. Thus, for each off-peak shift value, we define the effective signal as the difference between the expected value of the sample cross-correlation function at the off-peak shift and the expected peak value, as shown in Fig. 1. Thus, for a given true image $i$, we define the SNR at any shift $(z, w)$ as the ratio between the effective signal squared and the sum of the variance of the noise at $(z, w)$ and the variance of the noise at $(\alpha, \beta)$. We will use the above notion of "signal" and "noise," averaged over all possible images $i$, to define the figure-of-merit $F_{C}$ for the 2-D cross-correlation shift estimator. More precisely

$$
F_{C}(z, w, \alpha, \beta) \triangleq \frac{(\mathbf{E}[C(\alpha, \beta)]-\mathbf{E}[C(z, w)])^{2}}{\mathbf{E}[\operatorname{var}[C(\alpha, \beta) \mid i]+\operatorname{var}[C(z, w) \mid i]]}
$$

where the factors appearing in the denominator are conditional variances (e.g., $\operatorname{var}[C(\alpha, \beta) \mid i]=\mathbf{E}\left[C^{2}(\alpha, \beta) \mid i\right]-$ $\left.\mathbf{E}[C(\alpha, \beta) \mid i]^{2}\right)$ and the expectation in the denominator of (5) represents ensemble averaging of the image $i$. The conditional variances involve expectations conditioned on $i$. If noise were not present in the system, $F_{C}$ would be a function only of the differences $|(z-\alpha)|$ and $|(w-\beta)|$, but because of noise, whose 
correlation is not always dependent on these differences, the figure-of-merit is a function over $(z, w, \alpha, \beta)$. Similarly, for the projection-based shift estimator, we define the figure-of-merit in the vertical and horizontal directions, respectively, as

and

$$
F_{P_{y}}(z, \alpha, \beta) \triangleq \frac{\left(\mathbf{E}\left[P_{y}(\alpha)\right]-\mathbf{E}\left[P_{y}(z)\right]\right)^{2}}{\mathbf{E}\left[\operatorname{var}\left[P_{y}(z) \mid i\right]+\operatorname{var}\left[P_{y}(\alpha) \mid i\right]\right]}
$$

$$
F_{p_{x}}(w, \alpha, \beta) \triangleq \frac{\left(\left[\mathbf{E}\left[P_{x}(\beta)\right]-E\left[P_{x}(w)\right]\right)^{2}\right.}{\mathbf{E}\left[\operatorname{var}\left[P_{x}(w) \mid i\right]+\operatorname{var}\left[P_{x}(\beta) \mid i\right]\right]}
$$

In order to calculate the conditional variances in the above expressions, a sensor model must be chosen that relates the output data to the input image. Moreover, ensemble averaging over all images requires the selection of an appropriate statistical model for the image that suits specific applications.

\section{A. Sensor Model}

The sensor model examined in this paper describes the measurement as the scene intensity with the addition of both temporal and fixed-pattern noise. In particular, the noise terms $q_{1}(x, y)$ and $q_{2}(x, y)$ in (1) and (2), associated with the measurements $d_{1}$ and $d_{2}$, respectively, take the following form:

and

$$
q_{1}(x, y)=b(x, y)+n_{1}(x, y)
$$

$$
q_{2}(x, y)=b(x, y)+n_{2}(x, y) .
$$

The entries of the temporal noise matrices $n_{1}$ and $n_{2}$ are both spatially and temporally independent each with a variance equal to $\sigma^{2}$. On the other hand, the fixed-pattern noise term $b(x, y)$ is assumed to be temporally fixed between the two observed frames and its entries are assumed to be independent and identically distributed random variables each with a variance of $\sigma_{b}^{2}$. The fixed-pattern noise and the temporal noise are assumed mutually independent. According to this model, the expressions for the figures-of-merit given by (5), (6), and (7) reduce to $F_{c}(z, w, \alpha, \beta)=A_{c} / B_{c}, F_{P_{y}},(z, \alpha, \beta)=A_{P_{y}} / B_{P_{y}}$, and $F_{p_{x}}(w, \alpha, \beta)=A_{P_{x}} / B_{P_{x}}$, where

$$
\begin{aligned}
A_{c}= & \left(\sum _ { x = 1 } ^ { N } \sum _ { y = 1 } ^ { N } E \left[i^{2}(x, y)-i(x, y)\right.\right. \\
& \times i(z+x-\alpha, w+y-\beta)] \\
& \left.-N^{2} \sigma_{b}^{2}(\delta(z, w)-\delta(\alpha, \beta))^{2}\right) \\
B_{c}= & 4 \sum_{x=1}^{N} \sum_{y=1}^{N} E\left[i ( x , y ) \left(\left(\sigma^{2}+\sigma_{b}^{2}\right) i(x, y)\right.\right. \\
& \left.\left.+\sigma_{b}^{2} i(x-\alpha, y-\beta)\right)\right]+2 N^{2} \\
& \times\left(\sigma^{4}+\sigma_{b}^{4}(1+2 \delta(z, w))\right) \\
A_{P_{y}}= & \left(\sum_{x=1}^{N} \sum_{y=1}^{N} \sum_{y^{\prime}=1}^{N} E[i(x, y)(i(x, y-\beta)\right. \\
& \left.\left.-i\left(z+x-\alpha, y^{\prime}-\beta\right)\right)\right] \\
& \left.-N^{2} \sigma_{b}^{2}(\delta(z)-\delta(\alpha))^{2}\right)
\end{aligned}
$$

$$
\begin{aligned}
B_{P_{y}}= & 4 N \sum_{x=1}^{N} \sum_{y=1}^{N} \sum_{y^{\prime}=1}^{N} E\left[i ( x , y ) \left(\left(\sigma^{2}+\sigma_{b}^{2}\right)\right.\right. \\
& \left.\left.\times i\left(x, y^{\prime}-\beta\right)+\sigma_{b}^{2} i\left(x-\alpha, y^{\prime}-\beta\right)\right)\right] \\
& +2 N^{3}\left(\sigma^{4}+\sigma_{b}^{4}(1+2 \delta(z))\right) \\
A_{P_{x}}= & \left(\sum _ { x = 1 } ^ { N } \sum _ { y = 1 } ^ { N } \sum _ { x ^ { \prime } = 1 } ^ { N } E \left[i ( x , y ) \left(i\left(x^{\prime}-\alpha, y\right)\right.\right.\right. \\
& \left.\left.-i\left(x^{\prime}-\alpha, w+y-\beta\right)\right)\right] \\
& \left.-N^{2} \sigma_{b}^{2}(\delta(w)-\delta(\beta))\right)^{2}
\end{aligned}
$$

and

$$
\begin{aligned}
B_{P_{x}}= & 4 N \sum_{x=1}^{N} \sum_{y=1}^{N} \sum_{x^{\prime}=1}^{N} E\left[i ( x , y ) \left(\left(\sigma^{2}+\sigma_{b}^{2}\right)\right.\right. \\
& \left.\left.\times i\left(x^{\prime}-\alpha, y\right)+\sigma_{b}^{2} i\left(x^{\prime}-\alpha, y-\beta\right)\right)\right] \\
& +2 N^{3}\left(\sigma^{4}+\sigma_{b}^{4}(1+2 \delta(w))\right) .
\end{aligned}
$$

Clearly, the figures-of-merit $F_{c}, F_{P_{y}}$, and $F_{p_{x}}$ are functions of the variance of the temporal and fixed-pattern noise and the true image at the focal plane array $i$, appearing as the auto-correlation function $\mathbf{E}[i(x, y) i(s+x, t+y)]$. In the following subsections, we specialize these figures-of-merit for specific image models.

1) Uncorrelated Image Model: To capture the cases for which the image exhibits little or no spatial correlation, we will assume a correlation function of the form $\mathbf{E}[i(x, y) i(s+x, t+y)]=i_{o} \delta(s, t)$, An example of images that possess this property include astronomical images of unresolvable stars. With the above auto-correlation model, the expression for $F_{c}$ becomes

$$
\begin{aligned}
& F_{c}(z, w, \alpha, \beta) \\
& =\frac{\left[N^{2} i_{o}(1-\delta(z-\alpha, w-\beta))-N^{2} \sigma_{b}^{2}(\delta(z, w)-\delta(\alpha, \beta))\right]^{2}}{4 N^{2} i_{o}\left(\sigma^{2}+\sigma_{b}^{2}(1+\delta(\alpha, \beta))+4 N^{2}\left(\sigma^{4}+(1+2 \delta(z, w)) \sigma_{b}^{4}\right)\right.}
\end{aligned}
$$

If we choose the combination of integers $\alpha, \beta, z$, and $w$ that minimizes $F_{c}$ without choosing $(z, w)=(\alpha, \beta)$, we obtain the lower bound

$$
\begin{aligned}
& F_{c}(z, w, \alpha, \beta) \\
& \quad \geq \frac{\left(N^{2} i_{o}-N^{2} \sigma_{b}^{2}\right)^{2}}{4 N^{2} i_{o}\left(\sigma^{2}+2 \sigma_{b}^{2}\right)+4 N^{2}\left(\sigma^{4}+3 \sigma_{b}^{4}\right)}
\end{aligned}
$$

By choosing $(z, w)=(0,0)$ the numerator is minimized. Similarly, this choice makes the denominator as large as possible. To further minimize $F_{c}$, we can choose $\delta(\alpha, \beta)$ to be zero in the numerator and one in the denominator. Because our goal is to achieve a lower bound for $F_{c}$, this choice is valid. For the projection-based estimator, the vertical-direction figure-of-merit reduces to

$$
\begin{aligned}
& F_{P_{y}}(z, \alpha, \beta) \\
& =\frac{\left(N^{2} i_{o}(1-\delta(z-\alpha))-N^{2} \sigma_{b}^{2}(\delta(z)-\delta(\alpha))\right)^{2}}{4 N^{3} i_{o}\left(\sigma^{2}+\sigma_{b}^{2}(1+\delta(\alpha, \beta))+4 N^{3}\left(\sigma^{4}+(1+2 \delta(z)) \sigma_{b}^{4}\right)\right.}
\end{aligned}
$$


which can be maximized over $\alpha, \beta$, and $z$ to yield the upper bound

$$
F_{P_{y}}(z, \alpha, \beta) \leq \frac{\left(N^{2} i_{o}+N^{2} \sigma_{b}^{2}\right)^{2}}{4 N^{3} i_{o}\left(\sigma^{2}+\sigma_{b}^{2}\right)+4 N^{3}\left(\sigma^{4}+\sigma_{b}^{4}\right)} .
$$

Hence, by combining the above bounds and performing the appropriate manipulations and simplifications (found in the Appendix), we obtain a lower bound for the ratio $F_{c} / F_{P_{y}}$ under the condition that $i_{o}>2 \sigma_{b}^{2}$

$$
\frac{F_{c}(z, w, \alpha, \beta)}{F_{P_{y}}(z, \alpha, \beta)} \geq 1
$$

A similar argument also shows that $F_{c} \geq F_{p_{x}}$. Hence, we conclude that the 2-D cross-correlation shift estimator is superior to the projection-based shift estimator when the image lacks spatial correlation. This result is intuitive since whenever spatial averaging is performed on an astronomical image, there is very little signal to average for most pixels contain only noise.

\section{B. Image Models Exhibiting Spatial Correlation}

In most practical cases, images that are formed in the focal plane of imaging systems possess some type of spatial correlation. Examples include imaging extended astronomical objects. For purposes of analysis, we will assume a simple correlation model of the form

$$
E[i(x, y) i(s+x, t+y)]=i_{o}\left(\frac{N-|s|}{2 N}+\frac{N-|t|}{2 N}\right) .
$$

While this correlation function may not accurately model the auto-correlation of all images, it does serve to demonstrate how spatial correlation affects the performance of the projectionbased estimator relative to the 2-D correlation-based estimator. We first consider the case for which fixed-pattern noise is absent (i.e., $\sigma_{b}^{2}=0$ ). In this special case, the figures-of-merit $F_{c}$ and $F_{P_{y}}$ become

$$
F_{c}(z, w, \alpha, \beta)=\frac{N^{2} i_{o}^{2}(|z-\alpha|+|w-\beta|)^{2}}{16 N^{2} i_{o} \sigma^{2}+8 N^{2} \sigma^{4}}
$$

and

$$
F_{P_{y}}(z, \alpha, \beta)=\frac{N^{4} i_{o}^{2}(|z-\alpha|)^{2}}{16 N^{4} i_{o} \sigma^{2}-N^{3}|\beta| i_{o} \sigma^{2}+8 N^{3} \sigma^{4}} .
$$

In order to compare the performance of the 2-D cross-correlation shift estimator with that of the projection-based estimator in the horizontal direction, we assume that the vertical shift $w$ within $F_{c}$ is known, i.e., we set $w=\beta$ and obtain a version of $F_{c}$ that is a function over $z$ only. This assumption does not affect the computation of $F_{P_{y}}$ and allows us to compare the figures-of-merit of the two techniques for shift in one direction
TABLE I

Shift Estimator Performance For the 2-D Cross-CoRrelator (2-D) AND THE PROJECTION-BASED (1-D) ESTIMATORS WITH ONLY TEMPORAL NoISE AND No SPATIAL CORRELATION IN THE IMAGE. Note THAT THE IMAGE POSSESSES No SPATIAL CORRELATION IN THIS EXAMPLE

\begin{tabular}{c|c|c} 
Estimator & Peak SNR & Error in Pixels \\
\hline \hline 1-D & 10 & 0 \\
2-D & 10 & 0 \\
\hline 1-D & 9 & .1 \\
2-D & 9 & 0 \\
\hline 1-D & 6 & 6.2 \\
2-D & 6 & 2.9
\end{tabular}

without addressing the negative effects on $F_{c}$ caused by mis-registration in the other dimension. In this case, it can be shown that the ratio of the two figures-of-merit becomes

$$
\frac{F_{P_{y}}(z, \alpha, \beta)}{F_{c}(z, \alpha, \beta)}=\frac{N\left(2 i_{o} \sigma^{2}+\sigma^{4}\right)}{2 N i_{o} \sigma^{2}-|\beta| i_{o} \sigma^{2}+\sigma^{4}}
$$

from which it follows that $F_{P_{y}} / F_{c} \geq 1$ if $(N-1) \sigma^{4}+|\beta| i_{o} \sigma^{2} \geq 0$. Clearly, the ratio $F_{P_{y}} / F_{c}$ is always greater than unity. We therefore conclude that, in the absence of fixed-pattern noise, the projection-based shift estimator is superior to the 2-D cross-correlation shift estimator when estimating shifts in the horizontal direction. A similar conclusion can be made for the estimate of the vertical shift.

We now address the analysis of the sensor model that contains both temporal and fixed-pattern noise. As before we set $w=\beta$ in the expression for $F_{c}$. After some algebra, we obtain the two equations shown at the bottom of the page. We now choose the combination of $z, \alpha, \beta$ that minimizes the ratio $F_{P_{y}} / F_{c}$. (The delta functions can be eliminated from the figure-of-merit if we choose the values for them that make $F_{P_{y}}$ as small as possible and $F_{c}$ as large as possible.) After some algebra, we obtain the final result

$$
\begin{aligned}
& \frac{F_{P_{y}}(z, \alpha, \beta)}{F_{c}(z, \alpha, \beta)} \\
& \geq \frac{\left(i_{o}^{2}-4 \sigma_{b}^{2} i_{o}+4 \sigma_{b}^{2}\right)\left(2 i_{o} N \sigma^{2}+3 i_{o} N \sigma_{b}^{2}+N\left(\sigma^{4}+\sigma_{b}^{4}\right)\right)}{i_{o}^{2}\left(N i_{o}\left(\sigma^{2}+2 \sigma_{b}^{2}\right)-i_{o} \sigma_{b}^{2}+\sigma^{4}+3 \sigma_{b}^{4}\right)} .
\end{aligned}
$$

From the above bound we infer that if $i_{o} \geq 12 \sigma_{b}^{2}$, the ratio $F_{P_{y}} / F_{c}$ is always greater than one. The same conclusion holds for the ratio of $F_{p_{x}} / F_{c}$. This shows that the projection-based

and

$$
F_{c}(z, \alpha, \beta)=\frac{\left[i_{o} N|z-\alpha|-2 N^{2} \sigma_{b}^{2}(\delta(z, \beta)-\delta(\alpha, \beta))\right]^{2}}{16 i_{o} N^{2}\left(\sigma^{2}+2 \sigma_{b}^{2}\right)+8 N^{2}\left(\sigma^{4}+\sigma_{b}^{4}(1+2 \delta(z, \beta))-8 N(|\alpha|+|\beta|) i_{o} \sigma_{b}^{2}\right.}
$$

$$
F_{P_{y}}(z, \alpha, \beta) \geq \frac{\left(i_{o} N^{2}|z-\alpha|-2 N^{2} \sigma_{b}^{2}(\delta(z)-\delta(\alpha))\right)^{2}}{8 i_{o} N^{4}\left(\sigma^{2}+2 \sigma_{b}^{2}\right)+8 N^{3}\left(\sigma^{4}+\sigma_{b}^{4}(1+2 \delta(z))\right)} .
$$



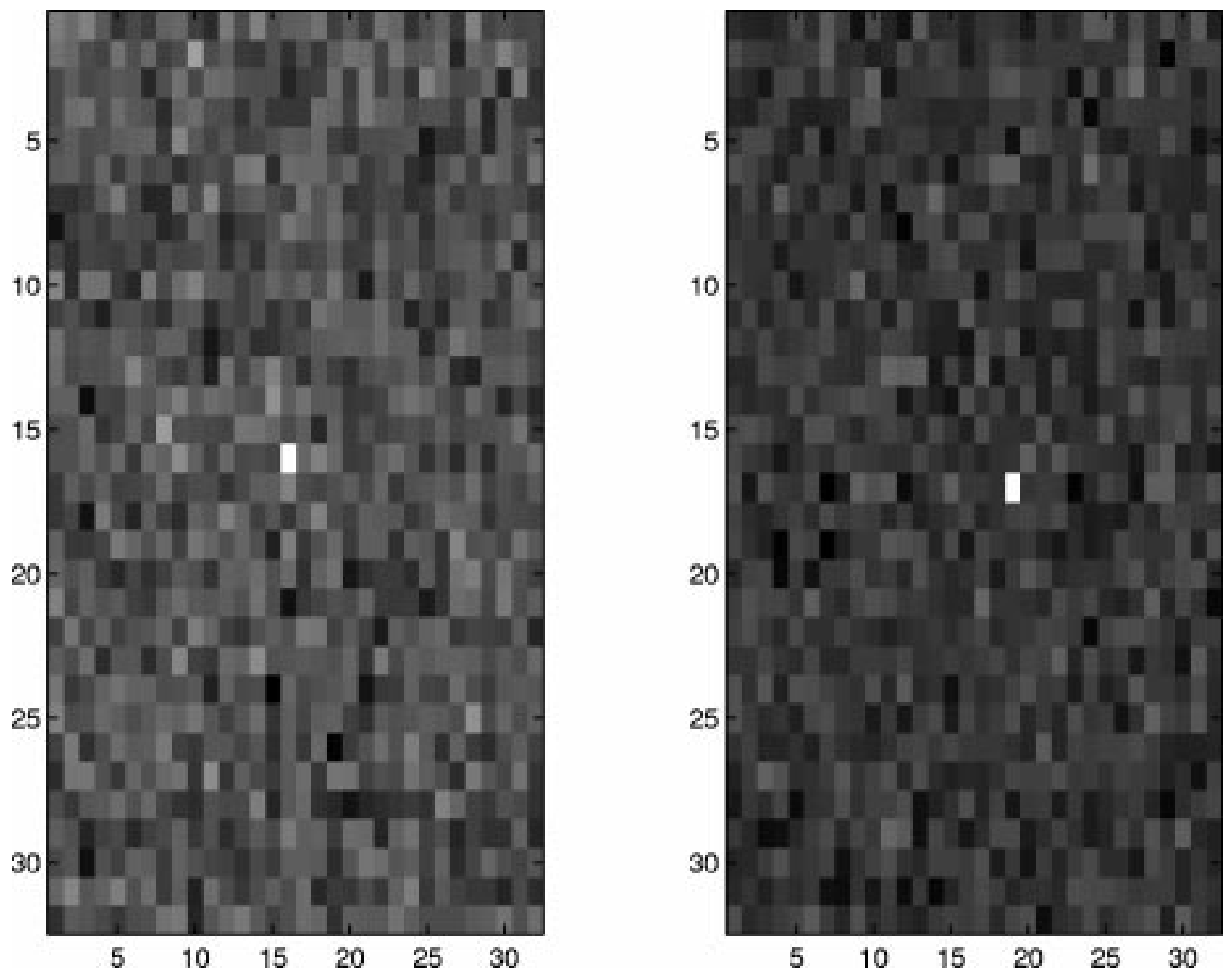

Fig. 2. Images showing a star in the presence of temporal noise at two different times.

shift estimator will have a higher SNR than the 2-D cross-correlation shift estimator whenever $i_{O}>12 \sigma_{b}^{2}$.

\section{Simulation Results}

In this section, a set of simulations is generated to demonstrate the validity of the conclusions established in the previous section. The first set of simulations feature an image of a simulated star field. This image exhibits no spatial correlation (approximating the uncorrelated image model considered in the previous section) and is corrupted with temporal Poissonian noise. (We use the Poisson model as a convenient approximation that captures the signal-dependent nature of temporal noise especially in cases when shot noise dominates the sensor read-out noise.) These images are shown in Fig. 2. The operation of generating two circularly shifted versions of the scene is repeated 100 times and shift estimates are generated using both the projection-based and 2-D correlation methods. These shift estimation algorithms are implemented exactly as described in Section II with a search range of seven pixels. The registration parameters $\alpha$ and $\beta$ are integers as are the shifts generated in the simulation. The cross-correlations are computed and the maximum is chosen over the entire range of shifts for both algorithms. Although real sequences of data generally possess noninteger and noncircular shifts, these assumptions are ade- quate for comparing the performance of the shift estimation techniques.

The shift estimates are compared with the known synthetic shifts for accuracy. The shift estimation error in pixels is computed by squaring the difference between the known synthetic shifts and the estimated shifts and summing this squared error over all frames. The result of this summation is then divided by the number of frames used in the test and the square root of this result is taken as the average error with units of pixels. The 100 trials are used to establish the statistical behavior of the shift estimation techniques. The results are shown in Table I. The performance of the two shift estimators are shown as a function of the SNR. The SNR is defined as the amplitude of the signal divided by the standard deviation of the noise. The peak signal-to-noise ratio (PSNR) in an image is then the highest SNR achieved within the frame. These results verify that indeed the 2-D cross-correlation shift estimator outperforms the projection-based shift estimator when the scene is spatially uncorrelated.

Next, a fixed-pattern noise is added to each frame and the standard deviation of the fixed-pattern noise $\sigma_{b}^{2}$ is varied in relation to the standard deviation of the temporal noise. Two sample images with fixed-pattern noise are shown in Fig. 3. The performance of the two estimators in the presence of fixed-pattern noise is shown in Table II averaged over 100 trials. The 

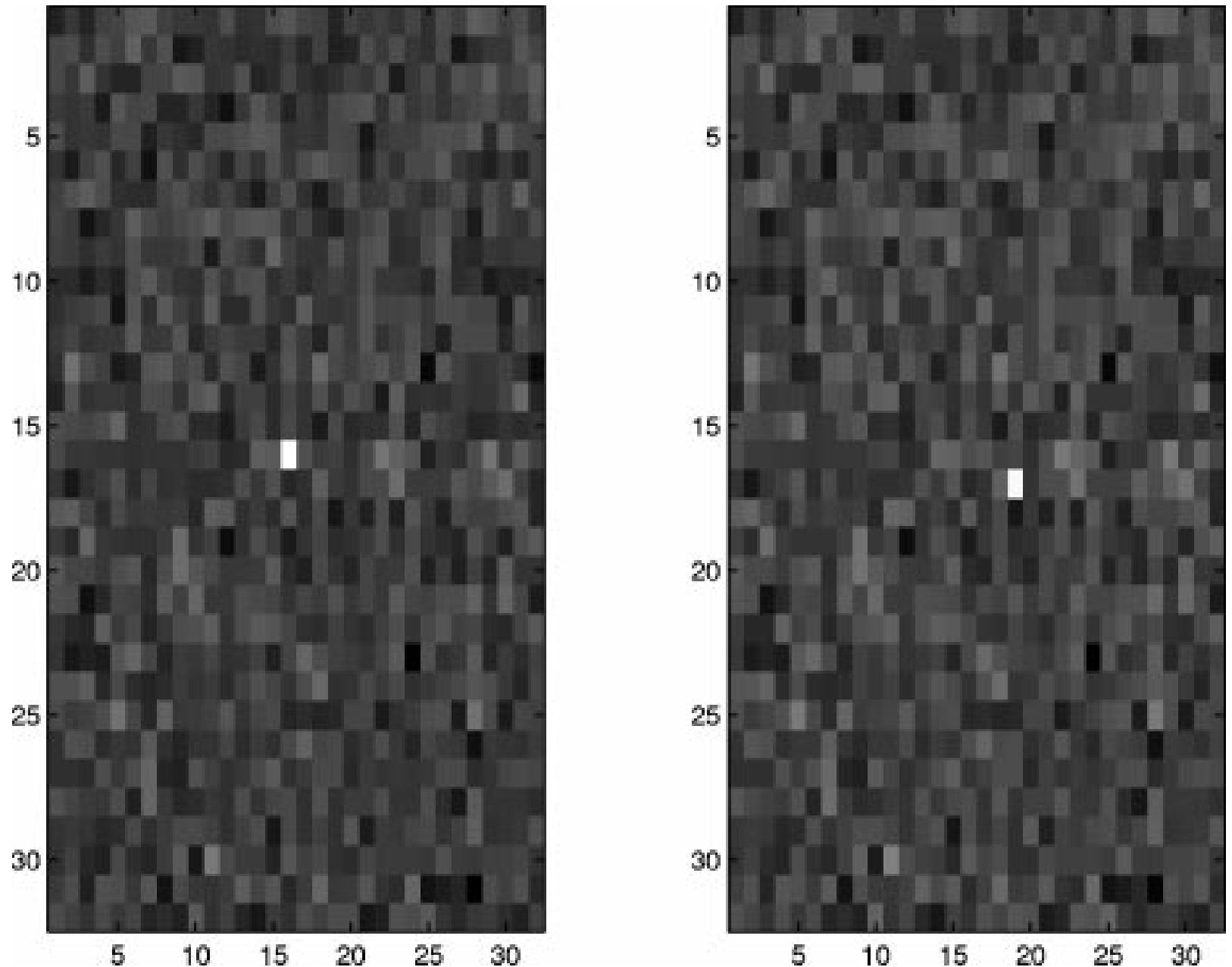

Fig. 3. Example of star images with fixed-pattern noise added to them.

PSNR of the data is fixed at ten and the ratio of fixed-pattern noise variance to temporal noise variance is varied. These results again verify that the lack of correlation in the scene causes the 2-D cross-correlation shift estimator to outperform the projection-based shift estimator. In general, we have observed that the performance of the two estimators becomes comparable as the fixed-pattern noise variance increases. The next set of data is generated in the same manner as in the previous simulations using the scene shown in Fig. 4, which possesses strong spatial correlation. This scene was chosen because its auto-correlation is similar to the linear auto-correlation model used in Section III. The auto-correlation function of the scene is shown in Fig. 5. The results, shown in Table III demonstrate that the projection-based shift estimator outperforms the 2-D cross-correlation shift estimator. Finally, Table IV shows the performance of the two estimators in the presence of fixed-pattern noise. The PSNR is fixed at 26 when the fixed-pattern noise is small with respect to the temporal noise. The fixed-pattern noise variance is adjusted to be a fraction of the temporal noise variance to produce the various ratios of fixed-pattern to temporal noise variance shown in Table IV. These results show that the performance improvement achieved using the projection-based shift estimator is more significant when fixed-pattern noise is a serious concern.

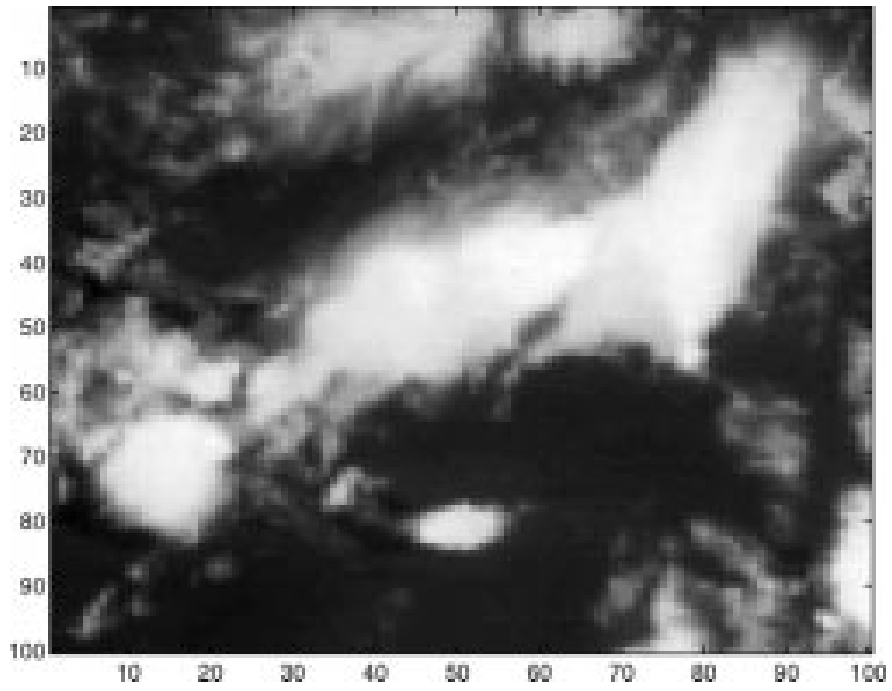

Fig. 4. Cloud scene used to generate the data used to analyze the behavior of the shift estimators in the presence of high spatial correlation.

To demonstrate the correlation between the simulations and the analytic expressions for the figures-of-merit, the magnitudes of $F_{P_{y}}$ and $F_{c}$ are plotted as a function of $\sigma_{b}\left(\sigma\right.$ and $i_{o}$ are held constant). If we choose $i_{o}=100, \sigma=10, \alpha=0, w=$ 


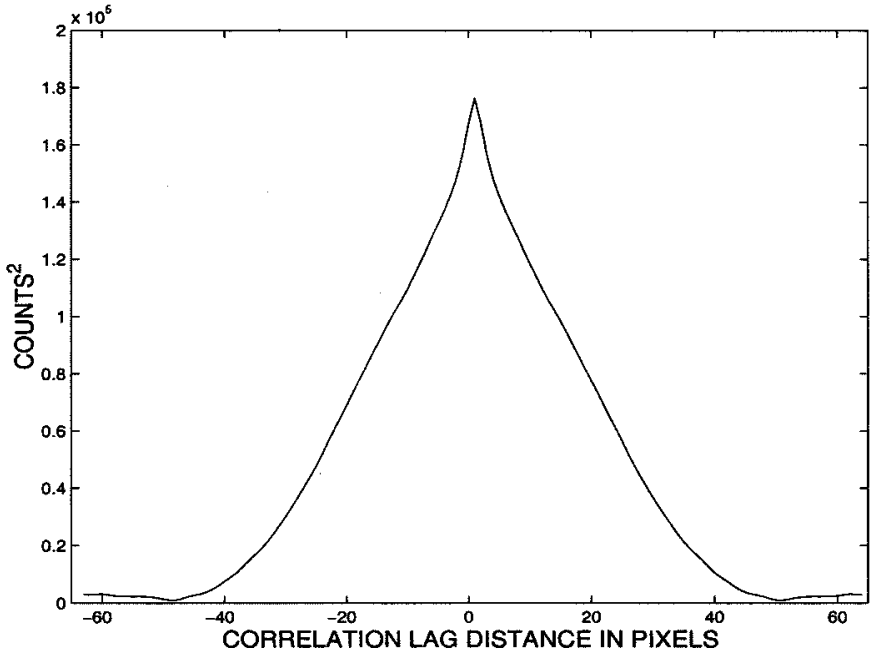

Fig. 5. Auto-correlation function of the scene used to test the performance of shift estimation in the presence of high spatial correlation.

TABLE II

Performance of the Projection-Based Estimator (1-D) Versus the 2-D Cross-Correlation ESTIMATOR (2-D) IN THE PRESENCE OF BOTH FiXed-PATTERn AND TeMPORAL NoISE. NOTE THAT THE IMAge POSSESSES No Spatial CORRELATION IN This EXAMPLE

\begin{tabular}{c|c|c} 
Estimator & $\sigma_{b} / \sigma$ & Error in Pixels \\
\hline \hline 1-D & .05 & .01 \\
$2-\mathrm{D}$ & .05 & 0 \\
\hline 1-D & .2 & .27 \\
2-D & .2 & .3 \\
\hline 1-D & .5 & .67 \\
2-D & .5 & .67
\end{tabular}

$\beta=0$, and $z=1$, then Fig. 6 shows the result of varying the fixed-pattern standard deviation $\sigma_{b}$. Note that over the whole range of fixed-pattern noise standard deviations the value of $F_{P_{y}}$ is higher than $F_{c}$.

\section{Results Using Real Image Sequences}

The proposed algorithm is tested on two sets of measured data in this section. Using measured data to test the accuracy of an image registration algorithm poses a distinct problem in that the true motion found in any real sequence of video is unknown without the use of a registration algorithm. In this analysis, we will measure the motion in a real sequence of data collected at a high SNR using the 2-D cross-correlator. This set of registration parameters are then used to measure error in both algorithms as noise is artificially added to the video. This process will allow the error in the shift estimation process to be measured using sequences of video that do not contain just simple translations, but other types of motion and effects that change the scene between observations.

The first sequence of video is taken with an infrared camera operating in the short-wave band (3.0-5.0 $\mu \mathrm{m}$ wavelengths).
TABLE III

Performance of the Projection-BASEd Estimator (1-D) Versus the 2-D CROSS-CORRELATION ESTIMATOR (2-D) WHEN SPATIAL CORRELATION IS PRESENT IN THE IMAGE

\begin{tabular}{c|c|c} 
Estimator & Peak SNR & Error in Pixels \\
\hline \hline 1-D & 26 & .1 \\
2-D & 26 & .3 \\
\hline 1-D & 10 & .2 \\
2-D & 10 & .45 \\
\hline 1-D & 7 & .45 \\
2-D & 7 & .5
\end{tabular}

TABLE IV

PERFormanCe OF THE PROJeCtion-BASEd Estimator (1-D) AND THE 2-D CROSS-CORRELATION ESTIMATOR (2-D) AGAINST TEMPORAL AND FiXed-PATtERn NOISE WhEN SPATIAL CORRELATION IS PRESENT IN THE IMAGE

\begin{tabular}{c|c|c} 
Estimator & $\sigma_{b} / \sigma$ & Error in Pixels \\
\hline \hline 1-D & .05 & .1 \\
2-D & .05 & .3 \\
\hline 1-D & 4 & .3 \\
2-D & 4 & .95 \\
\hline 1-D & 12 & .5 \\
2-D & 12 & 1.5
\end{tabular}

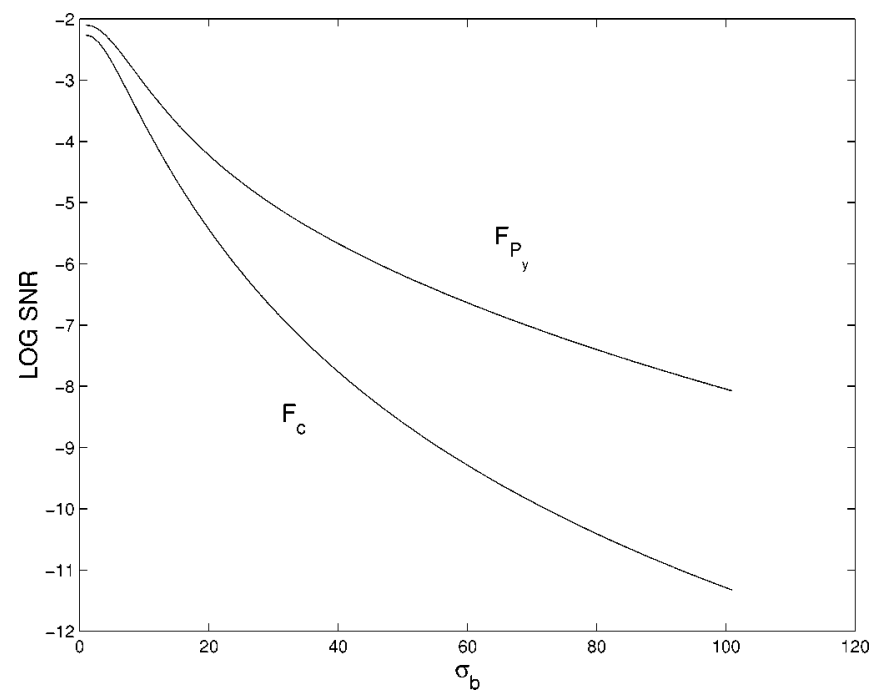

Fig. 6. Plot showing the value of the figures-of-merit $F_{P_{y}}$ and $F_{c}$ as a function of fixed-pattern noise standard deviation when $i_{o}=100, \sigma=10, \alpha=$ $0, w=\beta=0$, and $z=1$.

Two sample frames from this 80 -frame sequence are shown in Fig. 7. The 2-D cross-correlator is used to measure the shifts between frames of the sequence in integer amounts. This set of shifts is used as the true shifts for purposes of measuring the registration error when noise is added to the 

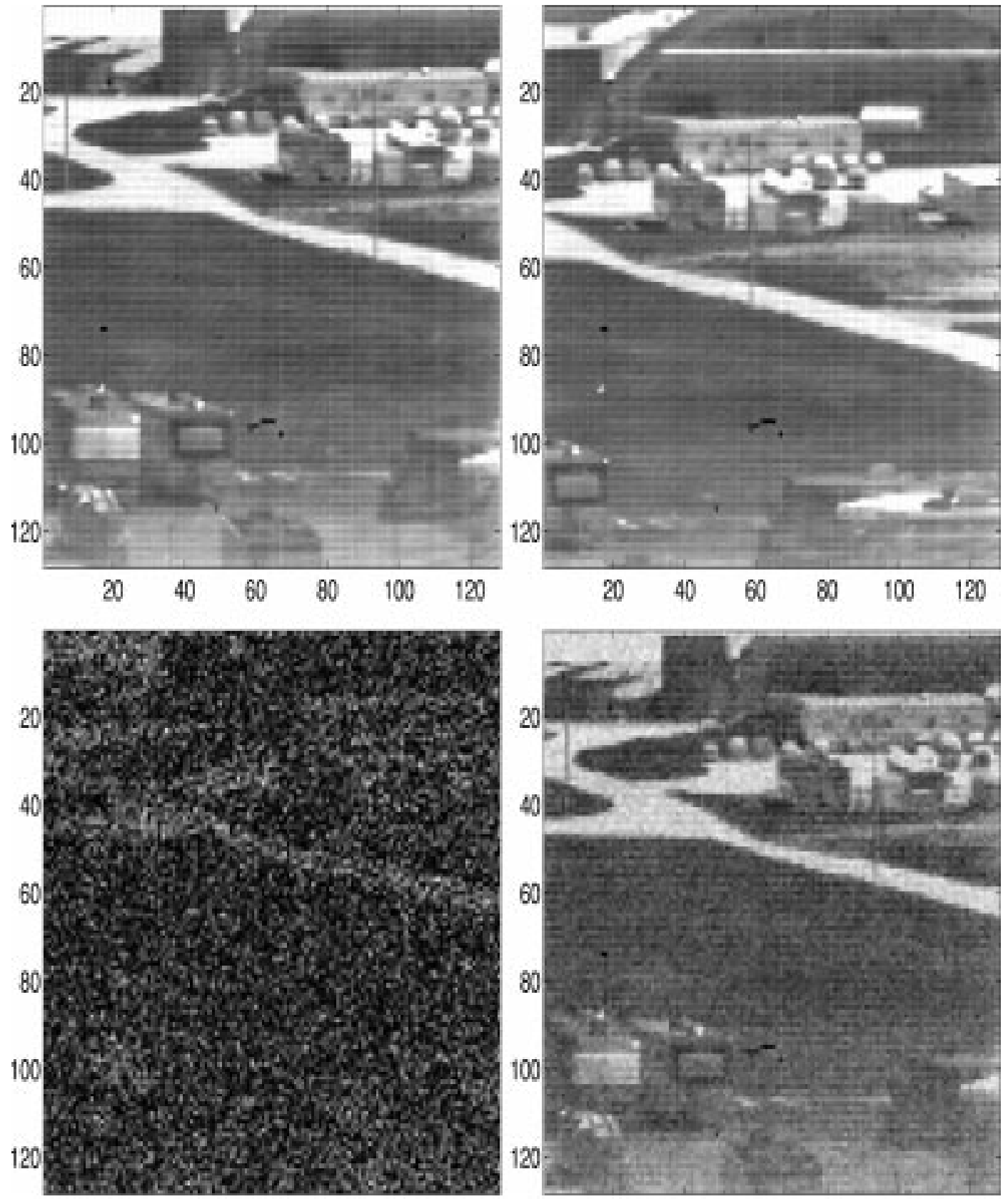

Fig. 7. Two sample frames from a sequence of infrared video data (top). Frame one of the sequence at a PSNR of 2 (bottom left) and PSNR of 20 (bottom right).

video sequence. In the first test, fixed-pattern noise is added to the sequence in varying amounts to produce 19 sequences each possessing a different PSNR between 2 and 20. Fig. 7 shows frame one of the sequence at a SNR of 2 and 20. Fig. 8 shows the error performance of the projection-based technique (dotted line) and the 2-D cross-correlator (solid line) plotted as a function of the PSNR in the data. The error metric used in this study is the root mean-squared error in units of pixels. Finally, Fig. 9 shows the performance of the algorithms as a function of the PSNR when the noise is temporal in nature (changes from frame to frame in the sequence). In both cases it is clear that the projection-based technique produces consistently lower registration root mean-squared error.

The second sequence of video is taken with a visible camera of the planet Jupiter through severe atmospheric turbulence.
This type of turbulence changes the impulse response of the imaging system between observations, which will cause the image to change in its appearance from frame to frame. Two sample frames from this 40 -frame sequence are shown in Fig. 10. The 2-D cross-correlator is again used to measure the shifts between frames of the sequence in integer amounts. In the first test, temporal noise is added to the sequence in varying amounts to produce ten video sequences each possessing a different PSNR between 1 and 10. Fig. 10 shows frame one of the sequence at a SNR of 1 and 10. Fig. 11 shows the error performance of the projection-based technique (dotted line) and the 2-D cross-correlator (solid line) plotted as a function of the peak temporal SNR in the data. Finally, Fig. 12 shows the performance of the algorithms as a function of the PSNR when fixed-pattern noise is added to the data. In both cases it is 


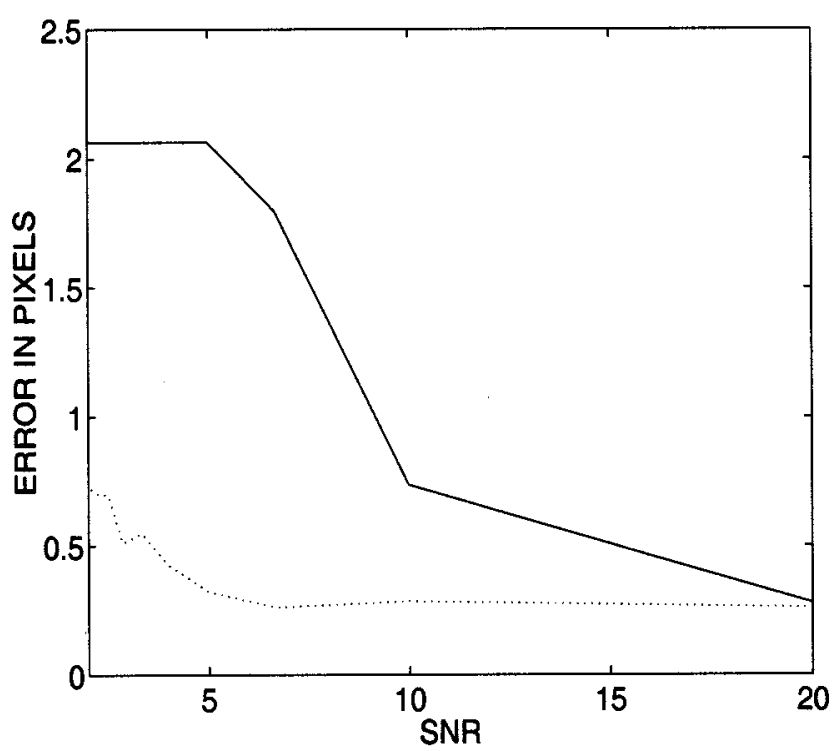

Fig. 8. Root mean-squared registration error performance of the projection-based technique (dotted line) and the 2-D cross-correlator (solid line) as a function of peak fixed-pattern SNR for the infrared data sequence. This demonstrates the improved noise-rejection capability of the projection-based technique.

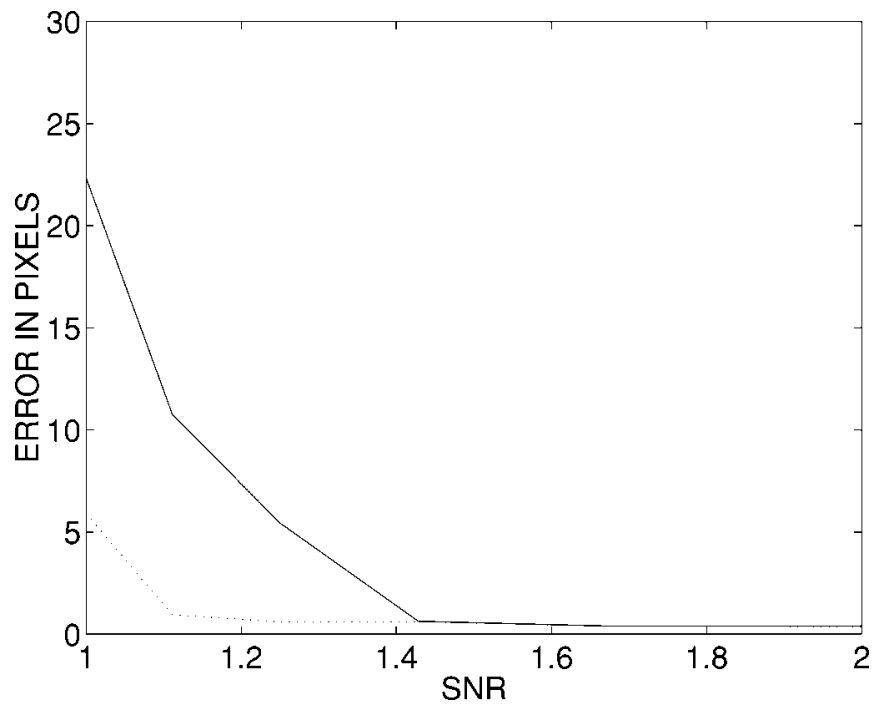

Fig. 9. Root mean-squared registration error performance of the projection-based technique (dotted line) and the 2-D cross-correlator (solid line) as a function of peak temporal SNR for the infrared data sequence.

clear that the projection-based technique produces consistently lower root mean-squared registration error.

\section{CONCLUSION}

Image registration of adjacent frames in a video sequence is often desired in order to take advantage of temporal correlation in images. This process can be very computationally intensive relative to the scale dictated by real time processing. The projection-based algorithm reported here has the potential of alleviating much of the computational burden of image registration by operating only on vectors as opposed to images. The proposed technique also has the benefit of being more robust against temporal and especially fixed-pattern noise, which can be a great impediment to achieving accurate image registration.
In this paper, the performance of the projection-based shift estimator is compared to the performance of the 2-D cross-correlation shift estimator. A significant conclusion drawn from this work is that the relative performance of the two estimators varies depending on the auto-correlation function of the scene.

The two auto-correlation models considered in the analysis show the diverse dependence of these algorithms on the degree of spatial correlation in the image. Scenes that possess significant neighbor-to-neighbor correlation over large neighborhoods will allow the projection-based shift estimator to perform better than the 2-D cross-correlator, while scenes whose pixels are uncorrelated allow the 2-D cross-correlator to produce superior results. Both estimators work well when the standard deviation of the noise is small with respect to the contrast in the scene. The decision of which estimator to use would be a simple one at high SNRs because the projection-based technique, as described in Section II, operates on $4 N$ pixels in comparison to $N^{2}$ pixels. The use of the 2-D cross-correlation technique is only warranted in cases when the SNR is extremely low and when the image lacks spatial correlation.

The potential applications for the projection-based algorithm are many and varied. Future work with the projection technique may involve adapting it for use with other geometric transformations such as rotation and scaling. The algorithm's tolerance to small rotations and scale changes has yet to be tested. Perhaps the most important application is in the field of real-time adaptive optics. This algorithm could be used to control a line-of-sight mirror within an optical system at kilohertz rates or provide the shift estimation information generated in Hartman sensors [20].

\section{APPENDIX \\ DERIVATION OF INEQUALITY (10)}

In this example, we assume a image auto-correlation function of the form $\mathbf{E}[i(x, y) i(s+x, t+y)]=i_{o} \delta(s, t)$. With this auto-correlation model, the expression for $F_{c}$ becomes

$$
\begin{aligned}
& F_{c}(z, w, \alpha, \beta) \\
& =\frac{\left[N^{2} i_{o}(1-\delta(z-\alpha, w-\beta))-N^{2} \sigma_{b}^{2}(\delta(z, w)-\delta(\alpha, \beta))\right]^{2}}{4 N^{2} i_{o}\left(\sigma^{2}+\sigma_{b}^{2}(1+\delta(\alpha, \beta))+4 N^{2}\left(\sigma^{4}+(1+2 \delta(z, w)) \sigma_{b}^{4}\right)\right.} .
\end{aligned}
$$

We wish to choose the combination of integer values of $\alpha, \beta, z$, and $w$ that minimizes $F_{c}$ without choosing $(z, w)=(\alpha, \beta)$. The condition that $(z, w) \neq(\alpha, \beta)$ arises from the definition of the figure-of-merit. The numerator of the figure-of-merit was defined as the peak of the image auto-correlation function minus the auto-correlation value of for some shift other than the peak value. The numerator measures the shift estimation algorithm's ability to distinguish the peak from other candidate shift values. If we evaluate the figure-of-merit for $(z, w)=(\alpha, \beta)$, then the numerator no longer measures the difference between the value at the correct shift and an erroneous shift and therefore defeats the purpose of using the figure-of-merit. So in minimizing the numerator subject to the constraint that $(z, w) \neq(\alpha, \beta)$, the impulse function $\delta(z-\alpha, w-\beta)$ goes to zero. The term $-N^{2} \sigma_{b}^{2}(\delta(z, w)-\delta(\alpha, \beta))$ is minimized if $\delta(z, w)$ is equal to one and $\delta(\alpha, \beta)$ is equal to zero. This is accomplished if $(z, w)=(0,0)$ and $(\alpha, \beta) \neq(0,0)$. Effecting these changes in 

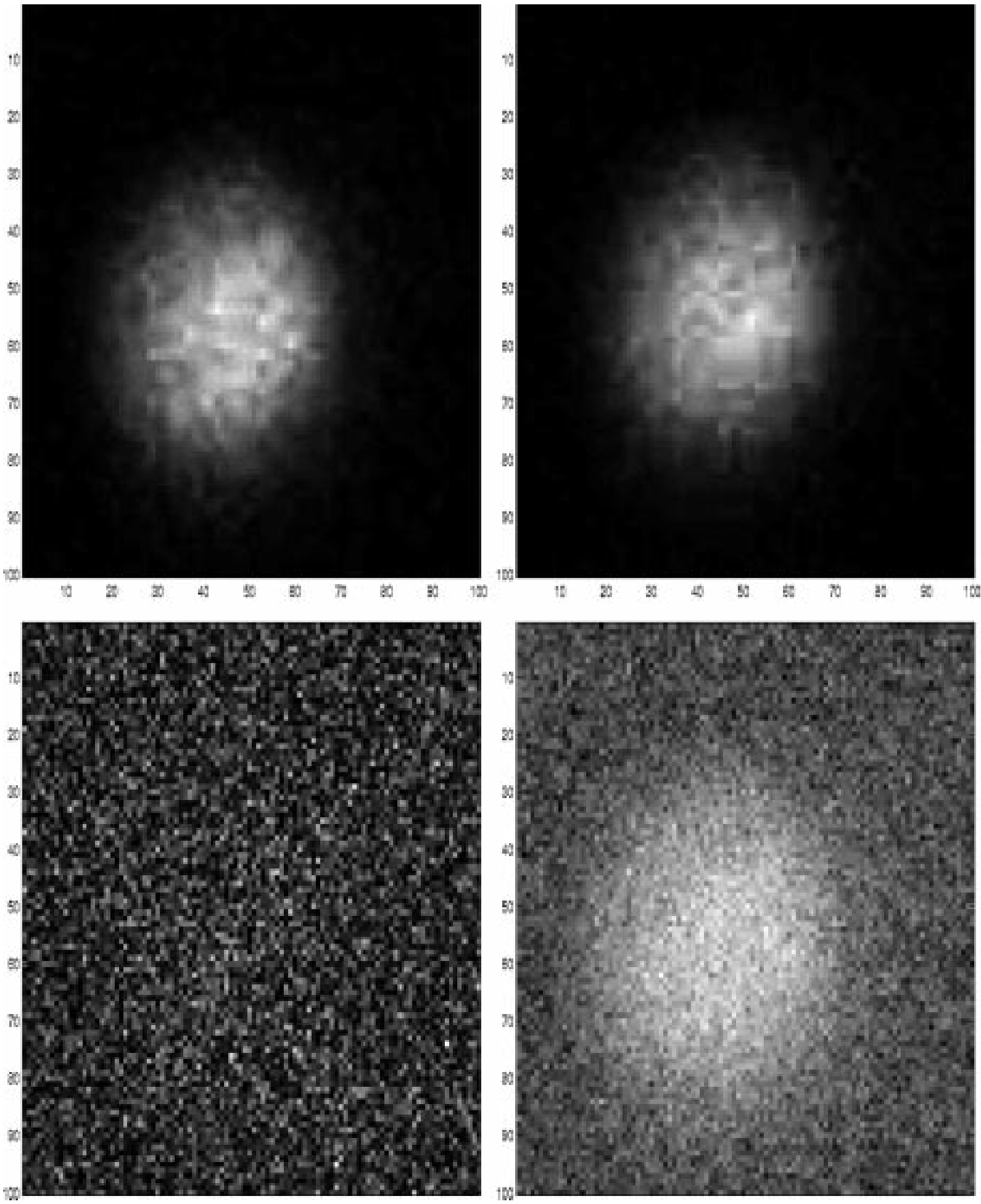

Fig. 10. (Top) Two sample frames from a sequence of video of the planet Jupiter, (bottom left) frame one of the sequence at a PSNR of 1, and (bottom right) PSNR of 10.

the numerator gives rise to the following inequality:

$$
\begin{aligned}
& F_{c}(z, w, \alpha, \beta) \\
& \geq \frac{\left(N^{2} i_{o}-N^{2} \sigma_{b}^{2}\right)^{2}}{4 N^{2} i_{o}\left(\sigma^{2}+\sigma_{b}^{2}(1+\delta(\alpha, \beta))+4 N^{2}\left(\sigma^{4}+(1+2 \delta(z, w)) \sigma_{b}^{4}\right)\right.}
\end{aligned}
$$

Next, we wish to maximize the denominator or determine an upper bound for it. Because all the terms in the denominator are strictly positive, if we choose them to be as large as possible, this will serve as an upper bound for the denominator. If all the impulse functions take on their maximum value, then the following inequality results:

$$
F_{c}(z, w, \alpha, \beta) \geq \frac{\left(N^{2} i_{o}-N^{2} \sigma_{b}^{2}\right)^{2}}{4 N^{2} i_{o}\left(\sigma^{2}+2 \sigma_{b}^{2}\right)+4 N^{2}\left(\sigma^{4}+3 \sigma_{b}^{4}\right)} .
$$




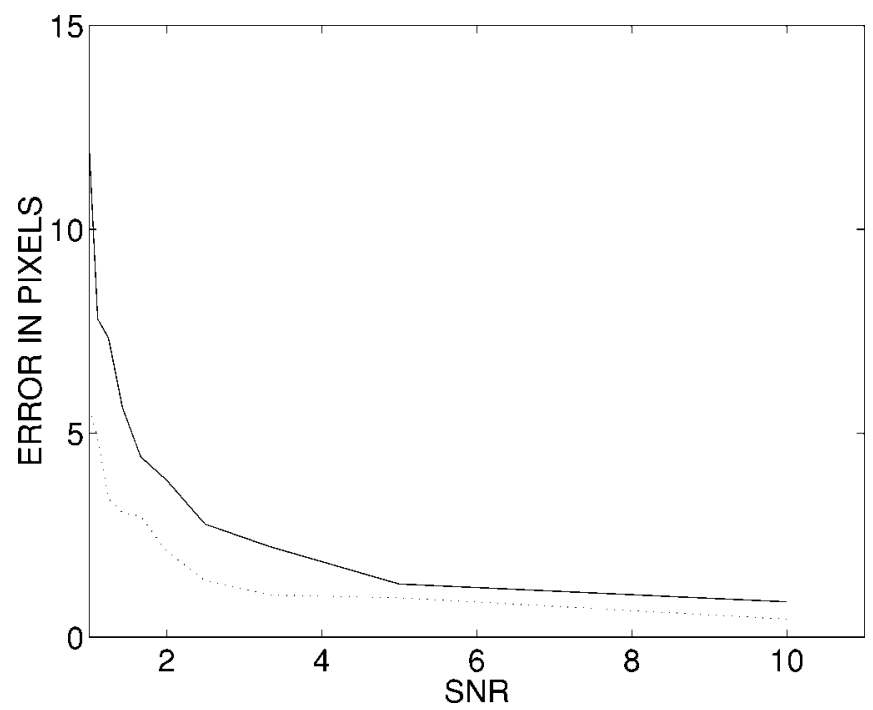

Fig. 11. Root mean-squared registration error performance of the projection-based technique (dotted line) and the 2-D cross-correlator (solid line) as a function of peak temporal SNR for the Jupiter data sequence.

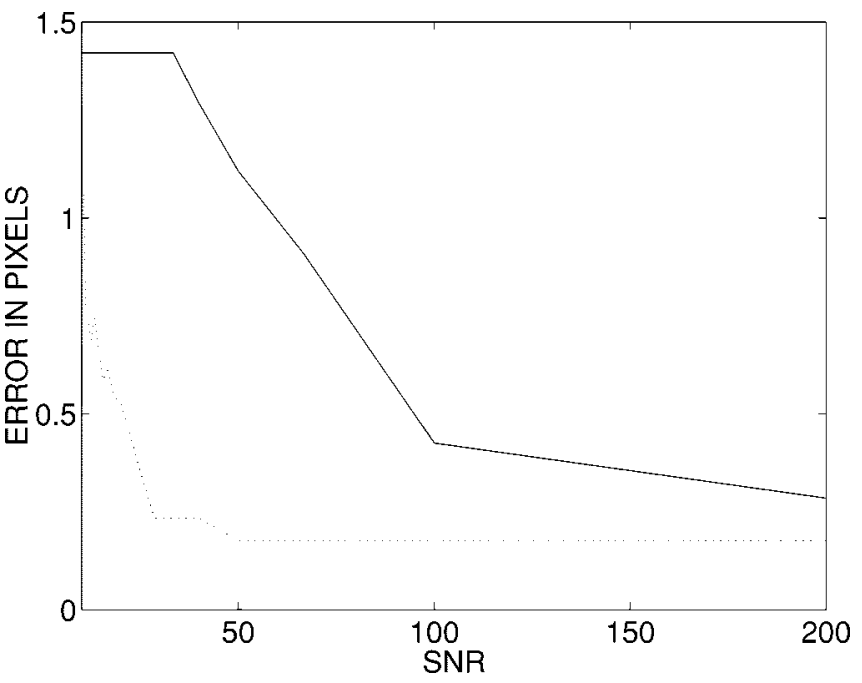

Fig. 12. Root mean-squared registration error performance of the projection-based technique (dotted line) and the 2-D cross-correlator (solid line) as a function of peak fixed-pattern SNR for the Jupiter data sequence.

For the projection-based estimator, the vertical-direction figure-of-merit can be computed and expressed as

$$
\begin{aligned}
& F_{P_{y}}(z, \alpha, \beta) \\
& \quad=\frac{\left(N^{2} i_{o}(1-\delta(z-\alpha))-N^{2} \sigma_{b}^{2}(\delta(z)-\delta(\alpha))\right)^{2}}{4 N^{3} i_{o}\left(\sigma^{2}+\sigma_{b}^{2}(1+\delta(\alpha, \beta))+4 N^{3}\left(\sigma^{4}+(1+2 \delta(z)) \sigma_{b}^{4}\right)\right.} .
\end{aligned}
$$

Applying the constraint for this figure-of-merit that $z \neq \alpha$, results in

$$
\begin{aligned}
& F_{P_{y}}(z, \alpha, \beta) \\
& \quad=\frac{\left(N^{2} i_{o}-N^{2} \sigma_{b}^{2}(\delta(z)-\delta(\alpha))\right)^{2}}{4 N^{3} i_{o}\left(\sigma^{2}+\sigma_{b}^{2}(1+\delta(\alpha, \beta))+4 N^{3}\left(\sigma^{4}+(1+2 \delta(z)) \sigma_{b}^{4}\right)\right.} .
\end{aligned}
$$

If we choose $\delta(z)=0$ and $\delta(\alpha)=1$, then the numerator is minimized. Because all the terms in the denominator are nonnegative, if we choose the impulse functions to be equal to zero, then this will serve as a lower bound for the value of the denominator. Now that the numerator is as large as possible and the denominator is as small as possible, the figure-of-merit is bounded above in the following inequality:

$$
F_{P_{y}}(z, \alpha, \beta) \leq \frac{\left(N^{2} i_{o}+N^{2} \sigma_{b}^{2}\right)^{2}}{4 N^{3} i_{o}\left(\sigma^{2}+\sigma_{b}^{2}\right)+4 N^{3}\left(\sigma^{4}+\sigma_{b}^{4}\right)} .
$$

Hence, by combining the above bounds, we obtain a lower bound for the ratio $F_{c} / F_{P_{y}}$

$$
\begin{aligned}
& \frac{F_{c}(z, w, \alpha, \beta)}{F_{P_{y}}(z, \alpha, \beta)} \\
& \geq \frac{N\left(i_{o}-\sigma_{b}^{2}\right)^{2}\left(i_{o}\left(\sigma^{2}+2 \sigma_{b}^{2}\right)+\sigma^{4}+3 \sigma_{b}^{4}\right)}{\left(i_{o}+\sigma_{b}^{2}\right)^{2}\left(i_{o}\left(\sigma^{2}+\sigma_{b}^{2}\right)+\sigma^{4}+\sigma_{b}^{4}\right)} .
\end{aligned}
$$

The term in the numerator $\left(i_{o}\left(\sigma^{2}+2 \sigma_{b}^{2}\right)+\sigma^{4}+3 \sigma_{b}^{4}\right)$ is always larger than the term $\left(i_{o}\left(\sigma^{2}+\sigma_{b}^{2}\right)+\sigma^{4}+\sigma_{b}^{4}\right)$. This means that the inequality can be reduced to the following expression:

$$
\frac{F_{c}(z, w, \alpha, \beta)}{F_{P_{y}}(z, \alpha, \beta)} \geq \frac{N\left(i_{o}-\sigma_{b}^{2}\right)^{2}}{\left(i_{o}+\sigma_{b}^{2}\right)^{2}} .
$$

If the ratio of the figures-of-merit is greater than one then the denominator subtracted from the numerator should be greater than zero

$$
(N-1)\left(i_{o}^{2}+\sigma_{b}^{4}\right)-(2 N+2) i_{o} \sigma_{b}^{2} \geq 0 .
$$

This inequality is always true if $(N-1) i_{o}>2(N+1) \sigma_{b}^{2}$. For large $N$, if $i_{o}>2 \sigma_{b}^{2}$ then $(N-1) i_{o}>2(N+1) \sigma_{b}^{2}$. This condition simply states that the average image intensity must be greater than twice the standard deviation of the fixed-pattern noise. Because the condition is met in most practical situations, $\left(F_{c}(z, w, \alpha, \beta)\right) /\left(F_{P_{y}}(z, \alpha, \beta)\right) \geq 1$.

\section{REFERENCES}

[1] S. C. Cain, E. Armstrong, and B. Yasuda, "Joint estimation of image, shift and nonuniformities from IR images," in Meeting of the IRIS Spe cialty Group on Passive Sensors 1, Tucson, AZ, 1997, pp. 121-132.

[2] M. Irani and S. Peleg, "Improving resolution by image registration," CVGIP Graph. Models Image Process., vol. 53, pp. 231-239, 1991.

[3] R. R. Schultz and R. L. Stevenson, "A Bayesian approach to image expansion for improved definition," IEEE Trans. Image Processing, vol. 3, no. 3, pp. 233-242, 1994.

[4] S. P. Kim, N. K. Bose, and H. M. Valenzuela, "Recursive reconstruction of high resolution image from noisy undersampled multiframes," IEEE Trans. Acoust., Speech, Signal Processing, vol. 38, pp. 1013-1027, June 1990.

[5] R. Y. Tsai and T. S. Huang, "Multi-frame image restoration and registration," Adv. Comput. Vis. Image Process., vol. 1, pp. 317-339, 1989.

[6] R. C. Hardie, M. M. Hayat, E. Armstrong, and B. Yasuda, "Scene based nonuniformity correction using video sequences and registration," Appl. Opt., vol. 39, no. 8, pp. 1241-1250, 2000.

[7] W. F. O'Neil, "Experimental verification of dithered scan nonuniformity correction," in Meeting of the IRIS Specialty Group on Passive Sensors 1, Tucson, AZ, 1997, pp. 329-339.

[8] E. Armstrong, M. M. Hayat, R. C. Hardie, S. Torres, and B. Yasuda, "The advantage of nonuniformity correction pre-processing on infrared image registration," Proc. SPIE, vol. 3808, pp. 150-161, 1999.

[9] G. C. Holst, CCD Arrays, Cameras, and Displays. Bellingham, WA: SPIE Optical Engineering Press, 1996.

[10] A. F. Milton, F. R. Barone, and M. R. Kruer, "Influence of nonuniformity on infrared focal plane array performance," Opt. Eng., vol. 24, no. 5, pp. 855-862, 1985.

[11] M. M. Hayat, S. N. Torres, E. Armstrong, S. C. Cain, and B. Yasuda, "Statistical algorithm for nonuniformity correction in focal-plane arrays," Appl. Opt., vol. 38, no. 8, pp. 772-780, 1999.

[12] D. I. Barnea and H. F. Silverman, "A class of algorithms for fast digital image registration," IEEE Trans. Comput., vol. C-21, no. 2, pp. 179-186, 1972. 
[13] A. Schaum and M. McHugh, "Analytic methods of image registration: Displacement estimation and resampling,", Naval Res. Rep. 9298, Feb. 1992.

[14] T. S. Huang, Image Sequence Analysis. Berlin, Germany: SpringerVerlag, 1981.

[15] S. C. Cain and K. D. Sauer, "Fast block motion estimation using integral projections," presented at the IEEE Workshop Visual Communications and Signal Processing, Raleigh, NC, 1992.

[16] K. Sauer and B. Schwartz, "Efficient block motion estimation using integral projections," IEEE Trans. Circuits Syst. Video Technol., vol. 6, pp. 513-518, Oct. 1996.

[17] J. S. Kim and R. H. Park, "A fast feature-based block matching algorithm using integral projections," IEEE J. Select. Areas Commun., vol. 10, pp. 968-971, June 1992.

[18] A. R. Hambley, Introduction to Communication Systems. Rockville, MD: Computer Science, 1990.

[19] H. Stark and J. W. Woods, Probability, Random Processes, and Estimation Theory for Engineers. Englewood Cliffs, NJ: Prentice-Hall, 1994.

[20] M. C. Roggemann and B. Welsh, Imaging Through Turbulence. Boca Raton, FL: CRC, 1996.

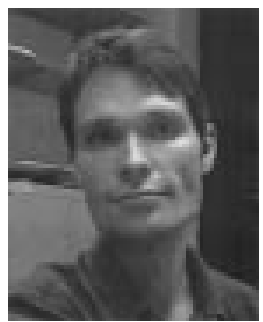

Stephen C. Cain was born in Pontiac, MI, in 1969. He received the B.S.E.E. degree from the University of Notre Dame, Notre Dame, IN, in 1992, the M.S.E.E. degree from Michigan Technological University, Houghton, in 1994, and the Ph.D. degree in electrical engineering from the University of Dayton, Dayton, $\mathrm{OH}$, in 2001.

From 1994 to 1997, he served as an Officer in the United States Air Force. From 1997 to 1998, he was with Wyle Laboratories and from 1998 to 2000, he was with ITT A/CD. In 2000, he began teaching as an Visiting Assistant Professor in the Department of Engineering, Purdue University at Fort Wayne, Fort Wayne, IN, where he now teaches as an Assistant Professor.

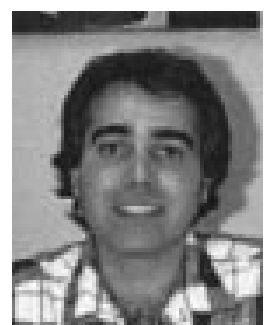

Majeed M. Hayat (S'89-M'92-SM'00) was born in Kuwait in 1963. He received the B.S. degree (summa cum laude) in electrical engineering from the University of the Pacific, Stockton, CA, in 1985, and the M.S. degree in electrical engineering and the $\mathrm{Ph} . \mathrm{D}$. degree in computer engineering, from the University of Wisconsin-Madison in 1988 and 1992, respectively.

From 1993 to 1996, he was with the University of Wisconsin-Madison as a Research Associate and Co-Principal Investigator of a project on statistical minefield modeling and detection, which was funded by the Office of Naval Research. In 1996, he joined the faculty of the Electro-Optics Graduate Program and the Department of Electrical and Computer Engineering, University of Dayton, Dayton, $\mathrm{OH}$, where he was granted early tenure and promotion to Associate Professor in 2000. He is currently an Associate Professor in the Department of Electrical and Computer Engineering at the University of New Mexico, Albuquerque. His research interests include modeling and design of high-performance photodetectors, optical communication systems, statistical communication theory, communication networks, infrared imaging, and statistical signal and image processing.

Dr. Hayat is a recipient of a 1998 National Science Foundation Early Faculty Career Award. He is a member of SPIE and OSA.

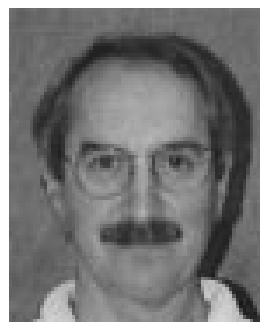

Ernest E. Armstrong received the B.S. degree in computer science and the M.S. degree in X-ray crystallography from Wright State University, Dayton, $\mathrm{OH}$, in 1977 and 1980, respectively, and received the $\mathrm{Ph} . \mathrm{D}$. degree in material analysis from Case Western Reserve University, Cleveland, $\mathrm{OH}$

$\mathrm{He}$ is currently a Senior Scientist (image processing) for OptiMetrics, Inc., a defense contractor at Wright Patterson AFB, OH. He previously held positions with Exxon Production Research and British Petroleum Research, where his research was primarily concerned with the application of signal and image processing techniques in materials analysis. He has authored and coauthored a number of publications in image processing, specializing in the area of image registration, resolution enhancement, and nonuniformity correction. 